Bull. Korean Math. Soc. 50 (2013), No. 2, pp. 389-398

http://dx.doi.org/10.4134/BKMS.2013.50.2.389

\title{
PURE INJECTIVE REPRESENTATIONS OF QUIVERS
}

\author{
Esmaeil Hosseini
}

\begin{abstract}
Let $R$ be a ring and $\mathcal{Q}$ be a quiver. In this paper we give another definition of purity in the category of quiver representations. Under such definition we prove that the class of all pure injective representations of $\mathcal{Q}$ by $R$-modules is preenveloping. In case $\mathcal{Q}$ is a left rooted semi-cobarren quiver and $R$ is left Noetherian, we show that every cotorsion flat representation of $\mathcal{Q}$ is pure injective. If, furthermore, $R$ is $n$-perfect and $\mathcal{F}$ is a flat representation $\mathcal{Q}$, then the pure injective dimension of $\mathcal{F}$ is at most $n$.
\end{abstract}

\section{Introduction}

Throughout the paper rings are associative with identity and modules are unital (unless otherwise specified). If $\mathcal{Q}$ is a quiver (a directed graph), then an arrow from a vertex $v_{1}$ to a vertex $v_{2}$ is denoted by $a: v_{1} \longrightarrow v_{2}$. The set of vertices (resp. arrows) of a quiver $\mathcal{Q}$ is denoted by $\mathrm{V}_{\mathcal{Q}}$ (resp. $\mathrm{E}_{\mathcal{Q}}$ ). For a given arrow $a$ of $\mathcal{Q}, i(a)$ denotes the initial vertex of $a$ and $t(a)$ denotes the terminal vertex of $a$. A quiver may be thought as a category in which the objects are vertices and the morphisms are paths, a path is a sequence of arrows. A representation $\mathcal{X}$ by modules of a quiver $\mathcal{Q}$ is then a covariant functor $\mathcal{X}: \mathcal{Q} \longrightarrow \mathrm{R}-$ Mod. Thus a representation $\mathcal{X}$ is determined by giving a module $\mathcal{X}(v)$ to each vertex $v \in \mathrm{V}_{\mathcal{Q}}$ and a homomorphism $\mathcal{X}(a): \mathcal{X}\left(v_{1}\right) \longrightarrow \mathcal{X}\left(v_{2}\right)$ to each arrow $a \in \mathrm{E}_{\mathcal{Q}}$. A morphism $f$ between representations $\mathcal{X}$ and $\mathcal{Y}$ is a natural transformation. The category of representations of a quiver $\mathcal{Q}$ by left $R$-modules over a ring $R$ is denoted by $\operatorname{Rep}(\mathcal{Q}, R)$. This is a Grothendieck category with projective generators and injective cogenerators (see [1] and [2]). A quiver $\mathcal{Q}$ is called left(right) rooted if there is no path of the form $\cdots \longrightarrow$ $\bullet \longrightarrow \bullet \longrightarrow \bullet(\bullet \longrightarrow \bullet \bullet \longrightarrow \cdots)$ in $\mathcal{Q}$. The study of special objects in the category of representations of quivers has a long history in the literature. Especially flat representations of a left rooted quiver, and existence of flat covers in the category $\operatorname{Rep}(\mathcal{Q}, R)$, when $\mathcal{Q}$ is left rooted, have been studied in [3], [8].

Received December 14, 2010; Revised May 15, 2012.

2010 Mathematics Subject Classification. Primary 16G20; Secondary 16E10.

Key words and phrases. representation of quiver, pure monomorphism, pure injective representation, cotorsion representation, flat representation, pure injective resolution.

This research was in part supported by a grant from IPM, No. 90130218. 
Let Flat $(\mathcal{Q})$ be the class of all flat representations of a left rooted quiver $\mathcal{Q}$ and $\operatorname{Flat}(\mathcal{Q})^{\perp}$, be the class of all object $\mathcal{C}$ of $\operatorname{Rep}(\mathcal{Q}, R)$ such that $\operatorname{Ext}_{\mathcal{Q}}^{1}(\mathcal{F}, \mathcal{C})=0$ for every $\mathcal{F} \in \operatorname{Flat}(\mathcal{Q})$. A representation $\mathcal{C}$ of $\mathcal{Q}$ is called cotorsion if $\mathcal{C} \in \operatorname{Flat}(\mathcal{Q})^{\perp}$. Therefore Flat $(\mathcal{Q})^{\perp}$ is the class of all cotorsion representations of $\mathcal{Q}$. Cotorsion representations of a left rooted quiver are characterized in [11].

Let us first recall some notations and results of [2, Corollary 6.7] that we need throughout. Let $\mathcal{Q}$ be a quiver. We can define the opposite quiver $\mathcal{Q}^{\text {op }}=$ $\left(\mathrm{V}_{\mathcal{Q}}, \mathrm{E}_{\mathcal{Q}}^{\mathrm{op}}\right)$ to $\mathcal{Q}$ such that its set of vertices is $\mathrm{V}_{\mathcal{Q}}$ and its set of arrows is $\mathrm{E}_{\mathcal{Q}}^{\mathrm{op}}$, in which $v \longrightarrow w \in \mathrm{E}_{\mathcal{Q}}^{\mathrm{op}}$ if and only if $w \longrightarrow v \in \mathrm{E}_{\mathcal{Q}}$. Note that $\operatorname{Rep}\left(\mathcal{Q}^{\mathrm{op}}, R^{\mathrm{op}}\right)$ is the category of representations of $\mathcal{Q}^{\text {op }}$ by right $R$-modules.

Let $\mathcal{X}$ be a representation of $\mathcal{Q}$, the representation $\mathcal{X}^{+} \in \operatorname{Rep}\left(\mathcal{Q}^{\text {op }}, R^{\text {op }}\right)$ is given by the following:

i) For any $v \in \mathrm{V}_{\mathcal{Q}}, \mathcal{X}^{+}=\operatorname{Hom}_{\mathbb{Z}}(\mathcal{X}(v), \mathbb{Q} / \mathbb{Z})$,

ii) For any $a \in \mathrm{E}_{\mathcal{Q}}$ such that $a: v \longrightarrow w, \mathcal{X}^{+}(a): \mathcal{X}^{+}(w) \longrightarrow \mathcal{X}^{+}(v)$.

Let $\operatorname{Inj}\left(\mathcal{Q}^{\text {op }}\right)$ be the class of all injective representations of $\mathcal{Q}^{\text {op }}$. By the following proposition, there is a fully faithful functor $\operatorname{Flat}(\mathcal{Q}) \stackrel{(-)^{+}}{\longrightarrow} \operatorname{Inj}\left(\mathcal{Q}^{\text {op }}\right)$.

Proposition 1.1. Let $\mathcal{Q}$ be a left rooted quiver. A representation $\mathcal{F}$ of $\mathcal{Q}$ is flat in $\operatorname{Rep}(\mathcal{Q}, R)$ if and only $\mathcal{F}^{+}$is an injective object of $\operatorname{Rep}\left(\mathcal{Q}^{\mathrm{op}}, R^{\mathrm{op}}\right)$.

Proof. See [2, Corollary 6.7].

The category $\operatorname{Rep}(\mathcal{Q}, R)$ is a locally finitely presented additive category, so there is a categorical notion of purity in terms of the finitely presented representations. By [9], every representation of $\mathcal{Q}$ has a pure injective envelope. Actually if $\mathcal{F}$ is a flat representation, it can be easily shown that the pure injective envelope (in the sense of [9]) is flat if, and only if, it coincides with its cotorsion envelope. Therefore the main result of this work, Theorem 3.6, can not be followed from [9]. So, for this end, we had to make a new definition of purity. We give many propositions to show that our notion of purity is wellbehaved. For example in Theorem 2.13 we prove that the classical relation between flatness and purity is true in $\operatorname{Rep}(\mathcal{Q}, R)$.

It is possible that the categorical notion of purity and our notion of purity are the same, but our notion of purity has some advantage. For instance, let $0 \longrightarrow \mathcal{X} \longrightarrow \mathcal{Y} \longrightarrow \mathcal{Z} \longrightarrow 0$ be an exact sequence of representations, then there exists the following commutative diagram with exact rows and pure exact columns

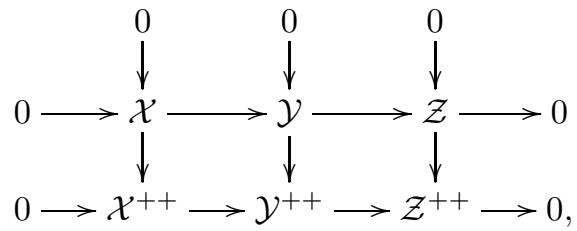

in $\operatorname{Rep}(\mathcal{Q}, R)$. But from the categorical notion of purity it is not clear if we can deduce this important diagram. 
In Section 2 we give the definition of purity in $\operatorname{Rep}(\mathcal{Q}, R)$ and study its properties, and show that under such definition each representation $\mathcal{X}$ of $\mathcal{Q}$ is a pure subrepresentation of a pure injective representation. In Section 3, over a left Noetherian ring we give necessary and sufficient conditions for $\mathcal{X} \in \operatorname{Rep}(\mathcal{Q}, R)$ to be flat and pure injective when $\mathcal{Q}$ is a left rooted semi-co-barren quiver. If $R$ is $n$-perfect, then the finiteness of the length of pure injective resolution of $\mathcal{X}$ will be discussed.

Setup: Throughout this paper $\mathcal{Q}$ is a left rooted quiver.

\section{Purity and pure injectivity}

This section is devoted to the study of purity in $\operatorname{Rep}(\mathcal{Q}, R)$. As a rich reference to the concepts of purity and pure injectivity and its properties in the category of $R$-modules, see [10], [12] and [13].

Definition 2.1. Let $\mathcal{A}$ be an abelian category and $\mathcal{C}$ be a class of objects of $\mathcal{A}$. For an object $\mathrm{A}$ of $\mathcal{A}$, an object $\mathrm{C} \in \mathcal{C}$ is called a $\mathcal{C}$-envelope of $A$ if there is a morphism $\varphi: \mathrm{A} \longrightarrow \mathrm{C}$ such that the following hold.

(i) For any morphism $\varphi^{\prime}: \mathrm{A} \longrightarrow \mathrm{C}^{\prime}$ with $\mathrm{C}^{\prime} \in \mathcal{C}$, there is a morphism $f: \mathrm{C} \longrightarrow \mathrm{C}^{\prime}$ with $\varphi^{\prime}=f \varphi$.

(ii) If an endomorphism $f: \mathrm{C} \longrightarrow \mathrm{C}$ is such that $\varphi=f \varphi$, then $f$ must be an automorphism.

If (i) holds, $\varphi: \mathrm{A} \longrightarrow \mathrm{C}$ is called a $\mathcal{C}$-preenvelope. Sometimes we call $\mathrm{C}$ or the map $\varphi$ a $\mathcal{C}$-envelope (preenvelope) of A. For more details on the concept of (pre)enveloping classes and their properties, see [6] and [12].

A morphism $f: \mathcal{X} \longrightarrow \mathcal{Y}$ of representations is called a monomorphism if $f$ has a cancelation property from the left, that is if for each representation $\mathcal{Z}$ of $\mathcal{Q}$ and each morphisms $g, h: \mathcal{Z} \longrightarrow \mathcal{X}$ which $f h=f g$, then $g=h$. On the other hand a morphism $f: \mathcal{X} \longrightarrow \mathcal{Y}$ is a monomorphism if and only if for every $v \in \mathrm{V}_{\mathcal{Q}}$, the morphism $f(v): \mathcal{X}(v) \longrightarrow \mathcal{Y}(v)$ is a monomorphism of $R$-modules. Now we make our definition of purity in $\operatorname{Rep}(\mathcal{Q}, R)$.

Definition 2.2. A monomorphism $f: \mathcal{X} \longrightarrow \mathcal{Y}$ in $\operatorname{Rep}(\mathcal{Q}, R)$ is a pure monomorphism if $f^{+}: \mathcal{Y}^{+} \longrightarrow \mathcal{X}^{+}$is a split epimorphism in the category $\operatorname{Rep}\left(\mathcal{Q}^{\mathrm{op}}, R^{\mathrm{op}}\right)$.

Remark 2.3. If $\mathcal{X} \longrightarrow \mathcal{Y}$ is a pure monomorphism in $\operatorname{Rep}(\mathcal{Q}, R)$, then $\mathcal{X}(v) \longrightarrow$ $\mathcal{Y}(v)$ is a pure monomorphism of $R$-modules for any vertex $v$ of $\mathcal{Q}$. But the converse is not true. To see this let $M$ be an arbitrary $R$-module. Consider the quiver $\mathcal{Q}: v_{1} \stackrel{a}{\longrightarrow} v_{2}$, and the representations $\mathcal{M}_{1}: M \stackrel{\mathcal{M}_{1}(a)}{\rightarrow} M \oplus M$ (where $\mathcal{M}_{1}(a)$ is injection) and $\mathcal{M}_{2}: M \oplus M \stackrel{\mathcal{M}_{2}(a)}{\longrightarrow} M \oplus M$ (where $\mathcal{M}_{2}(a)$ is identity). We see that $\mathcal{M}_{1}\left(v_{1}\right) \longrightarrow \mathcal{M}_{2}\left(v_{1}\right)$ and $\mathcal{M}_{1}\left(v_{2}\right) \longrightarrow \mathcal{M}_{2}\left(v_{2}\right)$ are pure monomorphisms of $R$-modules but $\mathcal{M}_{1} \stackrel{\theta}{\longrightarrow} \mathcal{M}_{2}$, where $\theta(a)=\left(\mathcal{M}_{1}(a), \mathcal{M}_{2}(a)\right)$, is not pure in $\operatorname{Rep}(\mathcal{Q}, R)$. 
Example 2.4. Let $\mathcal{X} \longrightarrow \mathcal{Y}$ be a monomorphism in $\operatorname{Rep}(\mathcal{Q}, R)$ such that for each vertex $v$ of $\mathcal{Q}, \mathcal{X}(v) \longrightarrow \mathcal{Y}(v)$ is a pure monomorphism of $R$-modules, and for each arrow $a: v \longrightarrow w, \mathcal{X}(a): \mathcal{X}(v) \longrightarrow \mathcal{X}(w)$ is a split epimorphism. Then $\mathcal{X} \longrightarrow \mathcal{Y}$ is a pure monomorphism in $\operatorname{Rep}(\mathcal{Q}, R)$.

Proposition 2.5. (i) Any split exact sequence

$$
0 \longrightarrow \mathcal{X} \longrightarrow \mathcal{Y} \longrightarrow \mathcal{Z} \longrightarrow 0
$$

in $\operatorname{Rep}(\mathcal{Q}, R)$ is pure exact.

(ii) Any direct limit of pure exact sequences is pure.

(iii) Let $\mathcal{X} \subseteq \mathcal{Y} \subseteq \mathcal{Z}$ be a sequence of subrepresentations of $\mathcal{Z}$. If $\mathcal{X}$ is a pure subrepresentation of $\mathcal{Z}$, then it is also pure as a subrepresentation of $\mathcal{Y}$.

Proof. (i) Since the exact sequence

$$
0 \longrightarrow \mathcal{X} \longrightarrow \mathcal{Y} \longrightarrow \mathcal{Z} \longrightarrow 0
$$

is split, $\mathcal{Y}=\mathcal{X} \oplus \mathcal{Z}$. Therefore $\mathcal{Y}^{+}=\mathcal{X}^{+} \oplus \mathcal{Z}^{+}$and so

$$
0 \longrightarrow \mathcal{Z}^{+} \longrightarrow \mathcal{Y}^{+} \longrightarrow \mathcal{X}^{+} \longrightarrow 0
$$

is split exact in $\operatorname{Rep}\left(\mathcal{Q}^{\text {op }}, R^{\text {op }}\right)$. Thus

$$
0 \longrightarrow \mathcal{X} \longrightarrow \mathcal{Y} \longrightarrow \mathcal{Z} \longrightarrow 0
$$

is pure exact sequence in $\operatorname{Rep}(\mathcal{Q}, R)$.

(ii) Let

$$
\left(0 \longrightarrow \mathcal{X}_{i} \longrightarrow \mathcal{Y}_{i} \longrightarrow \mathcal{Z}_{i} \longrightarrow 0\right)_{i \in I}
$$

be a direct system of pure exact sequences in $\operatorname{Rep}(\mathcal{Q}, R)$. Then

$$
\left(0 \longrightarrow \mathcal{Z}_{i}^{+} \longrightarrow \mathcal{Y}_{i}^{+} \longrightarrow \mathcal{X}_{i}^{+} \longrightarrow 0\right)_{i \in I}
$$

is an inverse system of split exact sequences in $\operatorname{Rep}\left(\mathcal{Q}^{\mathrm{op}}, R^{\mathrm{op}}\right)$. Therefore, for each $i \in \mathrm{I}$ we have the following split short exact sequence

$$
0 \longrightarrow \lim _{\longleftarrow} \mathcal{Z}_{i}^{+} \longrightarrow \lim _{\longleftarrow} \mathcal{Y}_{i}^{+} \longrightarrow \lim \mathcal{X}_{i}^{+} \longrightarrow 0
$$

and then we have the split short exact sequence

$$
\left.\left.0 \longrightarrow \underset{\overrightarrow{i \in I}}{\left(\lim _{i} \mathcal{Z}_{i}\right.}\right)^{+} \longrightarrow \underset{i \in I}{\left(\lim _{\vec{i}} \mathcal{Y}_{i}\right)^{+} \longrightarrow} \underset{\overrightarrow{i \in I}}{\left(\lim _{i} \mathcal{X}_{i}\right.}\right)^{+} \longrightarrow 0 .
$$

This implies that the short exact sequence

$$
0 \longrightarrow \underset{i \in I}{\lim } \mathcal{X}_{i} \longrightarrow \underset{i \in I}{\lim } \mathcal{Y}_{i} \longrightarrow \underset{i \in I}{\lim } \mathcal{Z}_{i} \longrightarrow 0,
$$

is pure exact sequence in $\operatorname{Rep}(\mathcal{Q}, R)$.

(iii) Let

$$
\mathcal{X} \hookrightarrow \mathcal{Y} \hookrightarrow \mathcal{Z}
$$


be a sequence of subrepresentations in $\operatorname{Rep}(\mathcal{Q}, R)$. We have the following commutative diagram in $\operatorname{Rep}(\mathcal{Q}, R)$ :

$$
\underset{\mathcal{Z}}{\mathcal{X} \stackrel{i_{2}}{\longrightarrow} \mathcal{Y}}
$$

Therefore we have the commutative diagram:

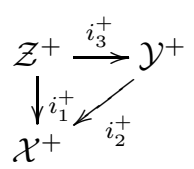

$\operatorname{Rep}\left(\mathcal{Q}^{\mathrm{op}}, R^{\mathrm{op}}\right)$, and there exists $k_{1}: \mathcal{X}^{+} \longrightarrow \mathcal{Z}^{+}$such that $i_{1}^{+} k_{1}=1_{X^{+}}$. So

$$
i_{2}^{+}\left(i_{3}^{+} k_{1}\right)=\left(i_{2}^{+} i_{3}^{+}\right) k_{1}=i_{1}^{+} k_{1}=1_{\mathcal{X}^{+}} .
$$

Thus the composition

is $1_{\mathcal{X}+}$. Therefore

$$
\mathcal{Y}^{+} \stackrel{i_{3}^{+}}{\longrightarrow} \mathcal{X}^{+} \stackrel{i_{2}^{+} k_{1}}{\longrightarrow} \mathcal{Y}^{+}
$$

$$
\mathcal{Y}^{+} \stackrel{i_{3}^{+}}{\longrightarrow} \mathcal{X}^{+}
$$

admits a section, and hence $\mathcal{X} \hookrightarrow \mathcal{Y}$ is a pure monomorphism in $\operatorname{Rep}(\mathcal{Q}, R)$.

Proposition 2.6. Let

$$
\mathcal{E}: 0 \longrightarrow \mathcal{X} \longrightarrow \mathcal{Y} \longrightarrow \mathcal{Z} \longrightarrow 0
$$

be an exact sequence in $\operatorname{Rep}(\mathcal{Q}, R)$.

(i) Let $\mathcal{E}$ be a pure exact sequence in $\operatorname{Rep}(\mathcal{Q}, R)$. Then $\mathcal{Y}$ is flat in $\operatorname{Rep}(\mathcal{Q}, R)$ if and only if $\mathcal{X}$ and $\mathcal{Z}$ are flat.

(ii) Let $\mathcal{Z}$ be a flat object of $\operatorname{Rep}(\mathcal{Q}, R)$. Then $\mathcal{X}$ is a flat object of $\operatorname{Rep}(\mathcal{Q}, R)$ if and only if $\mathcal{Y}$ is flat.

Proof. (i) Let $\mathcal{E}$ be a pure exact sequence of $\operatorname{Rep}(\mathcal{Q}, R)$. Then the exact sequence

$$
0 \longrightarrow \mathcal{Z}^{+} \longrightarrow \mathcal{Y}^{+} \longrightarrow \mathcal{X}^{+} \longrightarrow 0
$$

is split. So $\mathcal{Y}^{+}$is injective if and only if $\mathcal{X}^{+}$and $\mathcal{Z}^{+}$are injective. Therefore $\mathcal{Y}$ is a flat representation if and only if $\mathcal{X}$ and $\mathcal{Z}$ are flat.

(ii) The exact sequence

$$
0 \longrightarrow \mathcal{Z}^{+} \longrightarrow \mathcal{Y}^{+} \longrightarrow \mathcal{X}^{+} \longrightarrow 0
$$

is split. Thus

$$
\mathcal{Y}^{+}=\mathcal{X}^{+} \oplus \mathcal{Z}^{+}
$$

So $\mathcal{X}^{+}$is injective if and only if $\mathcal{Y}^{+}$is injective. Therefore $\mathcal{X}$ is flat if and only if $\mathcal{Y}$ is flat. 
Recall that a representation $\mathcal{Z}$ of $\mathcal{Q}$ is called pure injective if for any pure exact sequence

$$
0 \longrightarrow \mathcal{X} \longrightarrow \mathcal{Y}
$$

in $\operatorname{Rep}(\mathcal{Q}, R)$, the sequence

$$
\operatorname{Hom}_{\mathcal{Q}}(\mathcal{Y}, \mathcal{Z}) \longrightarrow \operatorname{Hom}_{\mathcal{Q}}(\mathcal{X}, \mathcal{Z}) \longrightarrow 0
$$

is exact in the category of abelian groups.

Proposition 2.7. Let $\mathcal{Q}$ be any quiver and $\mathcal{X}$ be a representation of $\mathcal{Q}$. Then

(i) The canonical monomorphism $\mathcal{X} \longrightarrow \mathcal{X}^{++}$is pure and $\mathcal{X}^{++}$is a pure injective representation.

(ii) A representation $\mathcal{X}$ is pure injective if and only if it is a direct summand of $\mathcal{X}^{++}$. Moreover, $\mathcal{Y}^{+}$is pure injective, for any representation $\mathcal{Y}$ of $\operatorname{Rep}\left(\mathcal{Q}^{\mathrm{op}}, R^{\mathrm{op}}\right)$.

Proof. In the first place we show that $\mathcal{X} \longrightarrow \mathcal{X}^{++}$is a pure injection. By definition, it suffices to give a section $\mathcal{X}^{+++} \longrightarrow \mathcal{X}^{+}$. But the canonical map $\mathcal{X}^{+} \longrightarrow \mathcal{X}^{+++}$does the job. Suppose then that $0 \longrightarrow \mathcal{N} \stackrel{i}{\longrightarrow} \mathcal{M}$ is a pure monomorphism and $f: \mathcal{N} \longrightarrow \mathcal{X}^{++}$is a map. Let $s: \mathcal{N}^{+} \longrightarrow \mathcal{M}^{+}$be such that $i^{+} s=1_{\mathcal{N}^{+}}$and set $g=s f^{+}: \mathcal{X}^{+++} \longrightarrow \mathcal{M}^{+}$. Clearly $g^{+} i^{++}=f^{++}$.

Note that since $\mathcal{X} \stackrel{\varphi \mathcal{X}}{\longrightarrow} \mathcal{X}^{++}$is a pure monomorphism, there exists a map $t: \mathcal{X}^{++++} \longrightarrow \mathcal{X}^{++}$which is a retraction for $\varphi_{\mathcal{X}}^{++}$, i.e., $t \varphi_{\mathcal{X}}^{++}=1_{\mathcal{X}++}$. On the other hand, since the canonical map $\varphi_{\mathcal{N}}$ is natural, we infer that $\varphi_{\mathcal{M}} i=i^{++} \varphi_{\mathcal{N}}$ and $f^{++} \varphi_{\mathcal{N}}=\varphi_{\mathcal{X}}^{++} f$. Now define $h=t g^{+} \varphi_{\mathcal{M}}$ and observe that $h i=f$. Thus the map $\operatorname{Hom}\left(\mathcal{M}, \mathcal{X}^{++}\right) \longrightarrow \operatorname{Hom}\left(\mathcal{N}, \mathcal{X}^{++}\right)$is a surjection and therefore $\mathcal{X}^{++}$ is pure injective.

(ii) Suppose $\mathcal{X}$ is a pure injective representation. Then from the canonical monomorphism $\varphi_{\mathcal{X}}$, which is pure by (i), one obtains a map $f: \mathcal{X}^{++} \longrightarrow \mathcal{X}$ satisfying $1_{\mathcal{X}}=f \varphi_{\mathcal{X}}$. The converse is obvious from (i). Meanwhile, since $\mathcal{X}^{+}$ is a summand of $\mathcal{X}^{+++}=\left(\mathcal{X}^{++}\right)^{+}$, we deduce that $\mathcal{X}^{+}$is pure injective.

Corollary 2.8. Every injective representation of $\mathcal{Q}$ is pure injective.

Proof. Let $\mathcal{I}$ be an injective representation of $\mathcal{Q}$. Then the canonical monomorphism $0 \longrightarrow \mathcal{I} \longrightarrow \mathcal{I}^{++}$is a split monomorphism and hence $\mathcal{I}$ is pure injective.

Remark 2.9. If $\mathcal{X}$ is a pure injective representation, then it possesses a pure injective $R$-module in each vertex. But the converse need not be true.

Example 2.10. If $\mathcal{X}$ is a representation of $\mathcal{Q}$ such that for any vertex $v$ of $\mathcal{Q}$, $\mathcal{X}(v)$ is pure injective and for any arrow $a$ of $\mathcal{Q}, \mathcal{X}(a)$ is a split epimorphism, then $\mathcal{X}$ is a pure injective representation of $\mathcal{Q}$.

Corollary 2.11. Every representation of $\mathcal{Q}$ has pure injective preenvelope. 
Proof. Let $\mathcal{X}$ be a representation of $\mathcal{Q}$ and $f: \mathcal{X} \longrightarrow \mathcal{Y}$ be a morphism of representations such that $\mathcal{Y}$ is pure injective. It is known that $\mathcal{X} \longrightarrow \mathcal{X}^{++}$ is a pure monomorphism. So there exists a morphism of representations $g$ : $\mathcal{X}^{++} \longrightarrow \mathcal{Y}$ such that the diagram

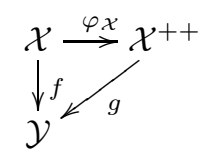

is commutative. This completes the proof.

Remark 2.12. By [11, Theorem 2.6], a representation $\mathcal{C}$ of $\mathcal{Q}$ is cotorsion if and only if it is cotorsion in each vertex. Therefore by Remark 2.9 every pure injective object of $\operatorname{Rep}(\mathcal{Q}, R)$ is a cotorsion representation of $\mathcal{Q}$.

Theorem 2.13. An object $\mathcal{Z}$ of $\operatorname{Rep}(\mathcal{Q}, R)$ is flat if and only if any exact sequence

$$
0 \longrightarrow \mathcal{X} \longrightarrow \mathcal{Y} \longrightarrow \mathcal{Z} \longrightarrow 0
$$

is pure in $\operatorname{Rep}(\mathcal{Q}, R)$.

Proof. If $\mathcal{Z}$ is flat in $\operatorname{Rep}(\mathcal{Q}, R)$, then for any exact sequence

$$
o \longrightarrow \mathcal{X} \longrightarrow \mathcal{Y} \longrightarrow \mathcal{Z} \longrightarrow 0
$$

in $\operatorname{Rep}(\mathcal{Q}, R)$, the sequence $0 \longrightarrow \mathcal{Z}^{+} \longrightarrow \mathcal{Y}^{+} \longrightarrow \mathcal{X}^{+} \longrightarrow 0$ is split exact in $\operatorname{Rep}\left(\mathcal{Q}^{\text {op }}, R^{\text {op }}\right)$, because $\mathcal{Z}^{+}$is injective. Hence (2.13.1) is pure.

Let every exact sequence

$$
0 \longrightarrow \mathcal{X} \longrightarrow \mathcal{Y} \longrightarrow \mathcal{Z} \longrightarrow 0
$$

be pure in $\operatorname{Rep}(\mathcal{Q}, R)$. It suffices to show that $\mathcal{Z}^{+}$is injective in $\operatorname{Rep}\left(\mathcal{Q}^{\text {op }}, R^{\text {op }}\right)$. For this end, let

$$
o \longrightarrow \mathcal{Z}^{+} \stackrel{f}{\longrightarrow} \mathcal{X} \longrightarrow \mathcal{Y} \longrightarrow 0
$$

be an exact sequence in $\operatorname{Rep}\left(\mathcal{Q}^{\mathrm{op}}, R^{\mathrm{op}}\right)$. Consider the following pullback diagram

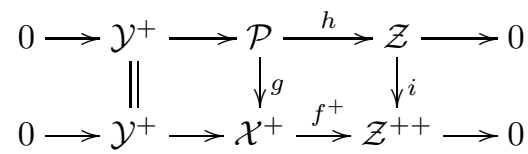

in $\operatorname{Rep}(\mathcal{Q}, R)$ with exact rows. By assumption the top row is pure and hence split, because $\mathcal{Y}^{+}$is pure injective. So there is a morphism $h^{\prime}: \mathcal{Z} \longrightarrow \mathcal{P}$ of representations such that $h h^{\prime}=1_{\mathcal{Z}}$. It follows that $g_{1}=g h^{\prime}: \mathcal{Z} \longrightarrow \mathcal{X}^{+}$ is a morphism of representations such that $f^{+} g_{1}=f^{+} g h^{\prime}=i h h^{\prime}=i$. Now 
consider the following commutative diagram

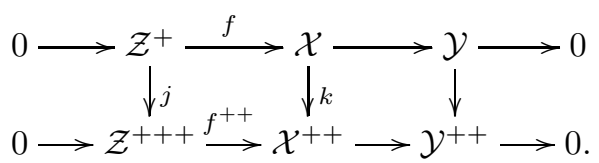

It follows that $g_{1}^{+} k f=g_{1}^{+} f^{++} j=i^{+} j=1_{\mathcal{Z}^{+}}$. Therefore (2.13.2) is split and hence $\mathcal{Z}^{+}$is an injective object of $\operatorname{Rep}\left(\mathcal{Q}^{\text {op }}, R^{\text {op }}\right)$. Then by Proposition $1.1, \mathcal{Z}$ is flat in $\operatorname{Rep}(\mathcal{Q}, R)$.

\section{Pure injective dimension of flat representations}

In this section we define the notion of a semi-co-barren quiver and give a characterization of a pure injective flat object in the category of representations of a semi-co-barren quiver.

Definition 3.1. A quiver $\mathcal{Q}$ is called semi-co-barren if for every $v \in \mathrm{V}_{\mathcal{Q}}$, $\left\{a \in \mathrm{E}_{\mathcal{Q}} \mid t(a)=v\right\}$ is a finite set.

Example 3.2. Let $\mathcal{Q}$ be a quiver whose connected components are barren trees. Then $\mathcal{Q}^{\text {op }}$ is a semi-co-barren quiver. Recall that a tree $T$ with a root $v$ is said to be barren if the number of vertices $n_{i}$ of the $i$ th state of $T$ is finite for every natural number $i$ and the sequence of positive natural numbers $n_{1}$, $n_{2}, \ldots$ stabilizes, for more details see [4] and [5].

Set up: In this section we let $R$ be a left Noetherian ring and $\mathcal{Q}$ be a semi-co-barren quiver or a quiver of type $A_{\infty}$ in the sense of [3].

Lemma 3.3. Let $\mathcal{F}$ be a representation of $\mathcal{Q}$. Then $\mathcal{F}$ is flat if and only if $\mathcal{F}^{++}$is a flat representation of $\mathcal{Q}$.

Proof. Let $\mathcal{F}$ be a flat representation of $\mathcal{Q}$. Since $\mathcal{Q}$ is a semi-co-barren quiver and $R$ is left Noetherian then for every $v \in \mathrm{V}_{\mathcal{Q}}, \bigoplus_{t(a)=v} \mathcal{F}^{++}(i(a)) \longrightarrow \mathcal{F}^{++}(v)$ is a split monomorphism of flat $R$-modules. Therefore by [8] and [3], $\mathcal{F}^{++}$is a flat representation of $\mathcal{Q}$.

The converse is a direct consequence of Proposition 2.6(i).

Theorem 3.4. Let $\mathcal{F}$ be a flat representation of $\mathcal{Q}$. Then the followings are equivalent:

(i) $\mathcal{F}$ is pure injective.

(ii) $\mathcal{F}$ is cotorsion.

(iii) $\mathcal{F}$ is isomorphic to a direct summand of $\mathcal{F}^{++}$.

Proof. (i) $\Rightarrow$ (ii) Let $\mathcal{F}$ be a pure injective representation of $\mathcal{Q} . \mathcal{F}$ is cotorsion in each vertex and hence by $[11$, Theorem 2.6] it is cotorsion object of $\operatorname{Rep}(\mathcal{Q}, R)$.

(ii) $\Rightarrow$ (iii) Let $\mathcal{F}$ be a cotorsion representation of $\mathcal{Q}$. By Lemma $3.3, \mathcal{F}^{++}$ and $\mathcal{F}^{++} / \mathcal{F}$ are flat representations of $\mathcal{Q}$. So

$$
0 \longrightarrow \mathcal{F} \stackrel{\lambda_{\mathcal{F}}}{\longrightarrow} \mathcal{F}^{++} \longrightarrow \operatorname{Coker} \lambda_{\mathcal{F}} \longrightarrow 0,
$$


is split and hence $\mathcal{F}$ is pure injective.

(iii) $\Rightarrow$ (i) This is trivial.

Corollary 3.5. Let $\mathcal{F}$ be a flat object of $\operatorname{Rep}(\mathcal{Q}, R)$. Then $\mathcal{F}$ is pure injective if and only if $\mathcal{F}(v)$ is pure injective $R$-module for all $v \in \mathrm{V}_{\mathcal{Q}}$.

Proof. Assume that for any $v \in \mathrm{V}_{\mathcal{Q}}, \mathcal{F}(v)$ is pure injective $R$-module. Thus for any $v \in \mathrm{V}_{\mathcal{Q}}, \mathcal{F}(v)$ is cotorsion. So by [11, Theorem 2.6], $\mathcal{F}$ is cotorsion object in $\operatorname{Rep}(\mathcal{Q}, R)$. Then by Theorem 3.4 it is pure injective.

The converse is trivial.

Let $\operatorname{Pinj}(\mathcal{Q})$ be the class of all pure injective objects in $\operatorname{Rep}(\mathcal{Q}, R)$. In Section 2 , we proved that $\operatorname{Pinj}(\mathcal{Q})$ is preenveloping. So every object $\mathcal{X}$ in $\operatorname{Rep}(\mathcal{Q}, R)$ has a unique(up to homotopy equivalence) pure injective resolution. Then for a given representation $\mathcal{X}$ of $\mathcal{Q}$, the pure injective dimension of $\mathcal{X}$ can be defined as follows

$$
\operatorname{pid} \mathcal{X}=\min \{n \mid \mathcal{X} \text { has a pure injective resolution of lenght } n\} .
$$

Recall that a ring $R$ is called $n$-perfect if for each flat $R$-module $F, \operatorname{cd} F$ (the cotorsion dimension of $F$ ) is at most $n$ (for more details see [7]). In the following theorem we show that, if $R$ is $n$-perfect, then

$$
\begin{aligned}
& \sup \{\operatorname{cd} \mathcal{F} \mid \text { for each flat representation } \mathcal{F}\} \\
= & \sup \{\operatorname{pid} \mathcal{F} \mid \text { for each flat representation } \mathcal{F}\} .
\end{aligned}
$$

Theorem 3.6. Let $R$ be an $n$-perfect ring and $\mathcal{F}$ be a flat representation of $\mathcal{Q}$. Then $\operatorname{pid} \mathcal{F} \leq n$.

Proof. Let $\mathcal{F}$ be a flat representation of $\mathcal{Q}$ and

$$
0 \longrightarrow \mathcal{F} \stackrel{\sigma}{\longrightarrow} \mathcal{C}^{0} \stackrel{\delta^{0}}{\longrightarrow} \mathcal{C}^{1} \stackrel{\delta^{1}}{\longrightarrow} \cdots \stackrel{\delta^{n-1}}{\longrightarrow} \mathcal{C}^{n} \stackrel{\delta^{n}}{\longrightarrow} \cdots,
$$

be a pure injective resolution of $\mathcal{X}$ such that

$$
\mathcal{C}^{i}=\left(\mathcal{C}^{i-1} / \operatorname{Im} \delta^{i-2}\right)^{++}
$$

for each $i \geq 2$. By Proposition 2.6(i) and Lemma 3.3, for each $i \geq 1$, Coker $\delta^{i-1}$ is a flat representation of $\mathcal{Q}$. Furthermore, for all $v \in \mathrm{V}_{\mathcal{Q}}$, the exact sequence

$$
0 \longrightarrow \mathcal{F}(v) \stackrel{\sigma(v)}{\longrightarrow} \mathcal{C}^{0}(v) \stackrel{\delta^{0}(v)}{\longrightarrow} \mathcal{C}^{1}(v) \stackrel{\delta^{1}(v)}{\longrightarrow} \cdots \stackrel{\delta^{n-1}(v)^{n}}{\longrightarrow} \mathcal{C}^{n}(v) \stackrel{\delta^{n}(v)}{\longrightarrow} \cdots,
$$

is a pure injective resolution of $\mathcal{F}(v)$ by pure injective flat $R$-modules. We know that for any $v \in \mathrm{V}_{\mathcal{Q}}, \operatorname{cd} \mathcal{F}(v) \leq n$. Then for any $v \in \mathrm{V}_{\mathcal{Q}}, \operatorname{Coker} \delta^{n-1}(v)$ is a cotorsion flat $R$-module. Therefore by [11, Theorem 2.6] $\operatorname{Coker} \delta^{n-1}$ is cotorsion flat, and by Theorem 3.4, it is pure injective flat in $\operatorname{Rep}(\mathcal{Q}, R)$. Then $\operatorname{pid} \mathcal{F} \leq n$.

Acknowledgements. The author is deeply grateful to the referee for his/her careful reading of the manuscript. I would like to thank the Institute for research in Fundamental Science(IPM). 


\section{References}

[1] E. Enochs and S. Estrada, Projective representations of quivers, Comm. Algebra 33 (2005), no. 10, 3467-3478.

[2] E. Enochs, S. Estrada, and J. R. Garcia Rozas, Injective representations of infinite quivers. Applications, Canad. J. Math. 61 (2009), no. 2, 315-335.

[3] E. Enochs, S. Estrada, J. Garcia Rozas, and L. Oyonarte, Flat covers of representations of the quiver $A_{\infty}$, Int. J. Math. Math. Sci. 2003 (2003), no. 70, 4409-4419.

[4] E. Enochs, S. Estrada, and S. Özdemir, Transfinite tree quivers and their representations, arXiv:1201.2294v1 [math.RA], (2012).

[5] E. Enochs, J. R. Garcia Rozas, L. Oyonarte, and S. Park, Noetherian quivers, Quaest. Math. 25 (2002), no. 4, 531-538.

[6] E. Enochs and O. Jenda, Relative Homological Algebra, Gordon and Breach S. Publishers, 2000

[7] E. Enochs, O. Jenda, and J. A. Lopez-Ramos, Dualizing modules and n-perfect rings, Proc. Edinb. Math. Soc. (2) 48 (2005), no. 1, 75-90.

[8] E. Enochs, L. Oyonarte, and B. Torrecillas, Flat covers and flat representations of quivers, Comm. Algebra 32 (2004), no. 4, 1319-1338.

[9] I. Herzog, Pure-injective envelope, J. Algebra Appl. 2 (2003), no. 4, 397-402.

[10] C. U. Jensen and H. Lenzing, Model Theoretic Algebra, Gordon and Breach S. Publishers, 1989.

[11] L. Oyonarte, Cotorsion representations of quivers, Comm. Algebra 29 (2001), no. 12, 5563-5574.

[12] J. Xu, Flat Covers of Modules, Lecture Notes in Mathematics, Springer-Verlag, 1634, 1996.

[13] R. Wisbauer, Foundations of Module and Ring Theory, Algebra, Logic and Applications, 3. Gordon and Breach Science Publishers, Philadelphia, PA, 1991.

Department of Mathematics

UNIVERSITY OF ISFAHAN

ISFAHAN, IRAN

E-mail address: e.hosseini@sci.ui.ac.ir 